\title{
Comparison of Connecting Rod Structural Analysis with Different Materials
}

\author{
N. Sreenivasa Babu \\ Engineering Department, \\ Shinas College of Technology, \\ Sultanate of Oman
}

\begin{abstract}
Connecting rod is an important link connects to the piston and crank shaft in transferring the power. Due to its importance, an attempt is made in evaluating the displacements, stresses and von-mises stress of the model under different loading conditions at crank end and pin end. Weight reduction is also carried to achieve the material savings by shape optimization and the analysis is continued. A typical model is chosen with $1.972 \%$ in weight reduction and a $2.53 \%$ increase in the von-mises stress due to shape optimization. The analyzed von-mises stresses are well in good understanding with the maximum limits of the material maximum stresses.
\end{abstract}

Key words: Von-mises stress, weight reduction, shape optimization, structural analysis.

\section{INTRODUCTION}

The crankshaft con-rod mechanism transforms reciprocative motion to rotational motion. The con-rod connects the piston to the crankshaft to transfer combustion pressure to the crankpin. There are bearing portions at both ends, the piston side is called the small end, and the crankshaft side, the big end. Connecting rods are commonly made from cast aluminum alloys to with stand dynamic stresses due from the combustion and piston movement. A typical focus is needed in evaluating the von-misses stress with different materials for comparison analysis. At instance, four different materials are chosen for the comparison purpose and properties are given from table:1.1.

Table: 1.1 Material properties

\begin{tabular}{|c|c|c|c|c|}
\hline S.No. & $\begin{array}{c}\text { Name of the } \\
\text { material }\end{array}$ & $\begin{array}{c}\text { Young's } \\
\text { Modulus, } \\
\mathrm{E} \\
\left(\mathrm{N} / \mathrm{mm}^{2}\right)\end{array}$ & $\begin{array}{c}\text { Density, } \\
\rho \\
\left(\mathrm{kg} / \mathrm{m}^{3}\right)\end{array}$ & $\begin{array}{c}\text { Poisson's } \\
\text { Ratio, } \\
1 / \mathrm{m}\end{array}$ \\
\hline 1 & Alloy steel & $2.1 \mathrm{e} 11$ & 7700 & 0.28 \\
\hline 2 & $\begin{array}{c}\text { Titanium } \\
\text { alloy, } \\
\text { Ti6al4V }\end{array}$ & $1.04 \mathrm{e} 11$ & 4429 & 0.31 \\
\hline 3 & $\begin{array}{c}\text { 1044 Carbon } \\
\text { steel }\end{array}$ & $2.05 \mathrm{e} 11$ & 7850 & 0.29 \\
\hline 4 & $\begin{array}{c}\text { Aluminum } \\
\text { alloy, A6061 }\end{array}$ & $0.69 \mathrm{e} 11$ & 2700 & 0.33 \\
\hline
\end{tabular}

\section{LITERATURE SURVEY}

Connecting rod is an important component that needs a typical analysis towards its structural stability under dynamic conditions [2] It is also critical to maintain an optimum weight to strength ratio to sustain inertial loads. Shape optimization is critically needed to reduce inertial load thus by minimizing the internal stresses during dynamic condition. Loading conditions are chosen [1] at the crank end and ping end to analyze the von-mises stresses and shape optimization is carried randomly to reduce the weight of the component. In the analysis, the authors emphasized on the calculation of the dimensions of the component and needs to be focused more on shape optimization and weight reduction. In this project, the shape optimization is chosen randomly and different materials like Alloy steel, Titanium alloy, Ti6al4V, 1044 Carbon steel and Aluminum alloy, A6061are chosen for structural analysis for the best suitable material with respect to cost and availability.

\section{MODEL DEVELOPMENT AND STRATEGY:}

A typical connecting rod is modeled using a AutoCAD software and imported in the analysis software Ansys $0[8]$ used to simulate engineering models to extract displacements and stresses. A solid brick element has chosen for its meshing element and different loading conditions (tension and compression) were considered at the crank end and pin end. At the crank end 10.11MPa in tension and 20.67 Mpa in compression is taken while other end is in fixed boundary condition. Similarly, at the pin end 22.24 MPa in tension and 20.17 Mpa in compression is taken and other end is in fixed boundary condition [1]. 


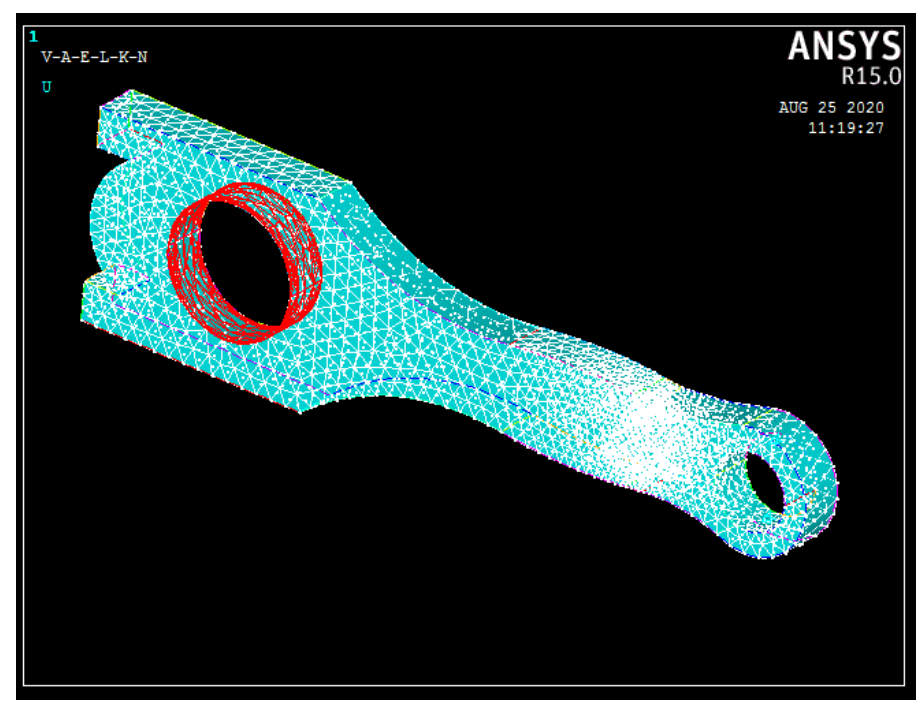

Fig: 3.1 A typical connecting rod model with loading conditions

\section{RESULTS AND DISCUSSIONS}

The connecting rod analysis is performed for its deformation, stress and von-mises stress with different materials under typical loading conditions. The results are tabulated and compared its dynamic stability.

Table 4.1 Tension loading at crank end $\left(10.11 \mathrm{~N} / \mathrm{mm}^{2}\right)$ on Fig: 3.1 model

\begin{tabular}{|c|c|c|c|c|c|c|}
\hline S.No. & $\begin{array}{c}\text { Name of the } \\
\text { material }\end{array}$ & $\begin{array}{c}\text { Deformation } \\
\text { in } \\
\text { X- direction } \\
(\mathrm{mm})\end{array}$ & $\begin{array}{c}\text { Deformation } \\
\text { in } \\
\text { Y-direction } \\
(\mathrm{mm})\end{array}$ & $\begin{array}{c}\text { Stress in } \\
\mathrm{X}- \\
\text { direction } \\
\left(\mathrm{N} / \mathrm{mm}^{2}\right)\end{array}$ & $\begin{array}{c}\text { Stress in } \\
\text { Y- } \\
\text { direction } \\
\left(\mathrm{N} / \mathrm{mm}^{2}\right)\end{array}$ & $\begin{array}{c}\text { Von- } \\
\text { mises } \\
\text { stress } \\
\left(\mathrm{N} / \mathrm{mm}^{2}\right)\end{array}$ \\
\hline 1 & Alloy steel & $0.66 \mathrm{e}-9$ & $0.30 \mathrm{e}-9$ & 22.90 & 24.42 & 28.05 \\
\hline 2 & $\begin{array}{c}\text { Titanium } \\
\text { alloy, } \\
\text { Ti6al4V }\end{array}$ & $0.13 \mathrm{e}-8$ & $0.61 \mathrm{e}-8$ & 22.99 & 24.82 & 28.07 \\
\hline 3 & $\begin{array}{c}1044 \\
\text { Carbon steel }\end{array}$ & $0.68 \mathrm{e}-9$ & $0.31 \mathrm{e}-8$ & 22.98 & 24.54 & 28.05 \\
\hline 4 & $\begin{array}{c}\text { Aluminum } \\
\text { alloy, } \\
\text { A6061 }\end{array}$ & $0.20 \mathrm{e}-8$ & $0.98 \mathrm{e}-8$ & 23.01 & 25.15 & 28.09 \\
\hline
\end{tabular}

Table 4.2 Compression loading at crank end (20.69 N/mm²) on Fig: 3.1 model

\begin{tabular}{|c|c|c|c|c|c|c|}
\hline S.No. & $\begin{array}{c}\text { Name of } \\
\text { the } \\
\text { material }\end{array}$ & $\begin{array}{c}\text { Deformation } \\
\text { in } \\
\text { X- direction } \\
(\mathrm{mm})\end{array}$ & $\begin{array}{c}\text { Deformation } \\
\text { in } \\
\text { Y- direction } \\
(\mathrm{mm})\end{array}$ & $\begin{array}{c}\text { Stress in } \\
\text { X- } \\
\text { direction } \\
\left(\mathrm{N} / \mathrm{mm}^{2}\right)\end{array}$ & $\begin{array}{c}\text { Stress in } \\
\text { Y- } \\
\text { direction } \\
\left(\mathrm{N} / \mathrm{mm}^{2}\right)\end{array}$ & $\begin{array}{c}\text { Von- } \\
\text { mises } \\
\text { stress } \\
\left(\mathrm{N} / \mathrm{mm}^{2}\right)\end{array}$ \\
\hline 1 & Alloy steel & $0.14 \mathrm{e}-9$ & $0.62 \mathrm{e}-9$ & 47.01 & 49.98 & 57.40 \\
\hline 2 & $\begin{array}{c}\text { Titanium } \\
\text { alloy, } \\
\text { Ti6al4V }\end{array}$ & $0.27 \mathrm{e}-8$ & $0.13 \mathrm{e}-7$ & 47.05 & 50.80 & 57.46 \\
\hline 3 & $\begin{array}{c}1044 \\
\text { Carbon } \\
\text { steel }\end{array}$ & $0.14 \mathrm{e}-8$ & $0.63 \mathrm{e}-8$ & 47.03 & 50.24 & 57.42 \\
\hline & $\begin{array}{c}\text { Aluminum } \\
\text { alloy, } \\
\text { A6061 }\end{array}$ & $0.41 \mathrm{e}-8$ & $0.19 \mathrm{e}-7$ & 47.08 & 51.46 & 57.51 \\
\hline
\end{tabular}

Table 4.3 Tension loading at pin end $\left(22.24 \mathrm{~N} / \mathrm{mm}^{2}\right)$ on Fig: 3.1 model

\begin{tabular}{|c|c|c|c|c|c|c|}
\hline S.No. & $\begin{array}{c}\text { Name of the } \\
\text { material }\end{array}$ & $\begin{array}{c}\text { Deformation in } \\
\text { X- direction } \\
(\mathrm{mm})\end{array}$ & $\begin{array}{c}\text { Deformation in } \\
\text { Y-direction } \\
(\mathrm{mm})\end{array}$ & $\begin{array}{c}\text { Stress in } \\
\mathrm{X}- \\
\text { direction } \\
\left(\mathrm{N} / \mathrm{mm}^{2}\right)\end{array}$ & $\begin{array}{c}\text { Stress in } \\
\text { Y- } \\
\text { direction } \\
\left(\mathrm{N} / \mathrm{mm}^{2}\right)\end{array}$ & $\begin{array}{c}\text { Von- } \\
\text { mises } \\
\text { stress } \\
\left(\mathrm{N} / \mathrm{mm}^{2}\right)\end{array}$ \\
\hline 1 & Alloy steel & $0.39 \mathrm{e}-8$ & $0.34 \mathrm{e}-8$ & 48.40 & 58.64 & 62.27 \\
\hline 2 & $\begin{array}{c}\text { Titanium } \\
\text { alloy, } \\
\text { Ti6al4V }\end{array}$ & $0.81 \mathrm{e}-8$ & $0.69 \mathrm{e}-8$ & 49.45 & 59.74 & 62.23 \\
\hline 3 & $\begin{array}{c}\text { 1044 Carbon } \\
\text { steel }\end{array}$ & $0.41 \mathrm{e}-8$ & $0.34 \mathrm{e}-8$ & 48.72 & 58.98 & 62.26 \\
\hline 4 & $\begin{array}{c}\text { Aluminum } \\
\text { alloy, A6061 }\end{array}$ & $0.12 \mathrm{e}-7$ & $0.10 \mathrm{e}-7$ & 50.31 & 60.93 & 62.21 \\
\hline
\end{tabular}


Table 4.4 Compression loading at pin end $\left(20.17 \mathrm{~N} / \mathrm{mm}^{2}\right)$ on Fig: 3.1 model

\begin{tabular}{|c|c|c|c|c|c|c|}
\hline S.No. & $\begin{array}{c}\text { Name of the } \\
\text { material }\end{array}$ & $\begin{array}{c}\text { Deformation } \\
\text { in } \\
\text { X-direction } \\
(\mathrm{mm})\end{array}$ & $\begin{array}{c}\text { Deformation } \\
\text { in } \\
\text { Y- direction } \\
(\mathrm{mm})\end{array}$ & $\begin{array}{c}\text { Stress in } \\
\mathrm{X}- \\
\text { direction } \\
\left(\mathrm{N} / \mathrm{mm}^{2}\right)\end{array}$ & $\begin{array}{c}\text { Stress in } \\
\text { Y- } \\
\text { direction } \\
\left(\mathrm{N} / \mathrm{mm}^{2}\right)\end{array}$ & $\begin{array}{c}\text { Von- } \\
\text { mises } \\
\text { stress } \\
\left(\mathrm{N} / \mathrm{mm}^{2}\right)\end{array}$ \\
\hline 1 & Alloy steel & $0.39 \mathrm{e}-8$ & $0.34 \mathrm{e}-8$ & 48.40 & 58.64 & 62.27 \\
\hline 2 & $\begin{array}{c}\text { Titanium } \\
\text { alloy, } \\
\text { Ti6al4V }\end{array}$ & $0.81 \mathrm{e}-8$ & $0.69 \mathrm{e}-8$ & 49.45 & 59.74 & 62.23 \\
\hline 3 & $\begin{array}{c}\text { 1044 Carbon } \\
\text { steel }\end{array}$ & $0.41 \mathrm{e}-8$ & $0.34 \mathrm{e}-8$ & 48.72 & 58.98 & 62.26 \\
\hline 4 & $\begin{array}{c}\text { Aluminum } \\
\text { alloy, } \\
\text { A6061 }\end{array}$ & $0.12 \mathrm{e}-7$ & $0.10 \mathrm{e}-7$ & 50.31 & 60.93 & 62.21 \\
\hline
\end{tabular}

The model is shape optimized to achieve weight reduction and the results are tabulated.

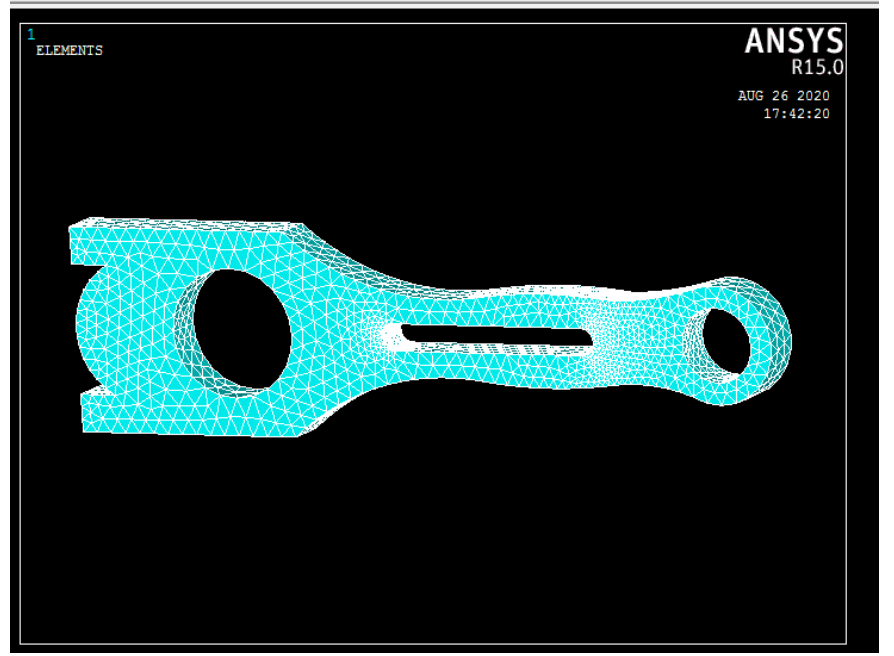

Fig: 4.1 A typical connecting rod model after shape optimization

Table 4.5 Tension loading at crank end $\left(10.11 \mathrm{~N} / \mathrm{mm}^{2}\right)$ on Fig: 4.1 model

\begin{tabular}{|c|c|c|c|c|c|c|}
\hline S.No. & $\begin{array}{c}\text { Name of the } \\
\text { material }\end{array}$ & $\begin{array}{c}\text { Deformation in } \\
\text { X-direction } \\
(\mathrm{mm})\end{array}$ & $\begin{array}{c}\text { Deformation in } \\
\text { Y-direction } \\
(\mathrm{mm})\end{array}$ & $\begin{array}{c}\text { Stress in X- } \\
\text { direction } \\
\left(\mathrm{N} / \mathrm{mm}^{2}\right)\end{array}$ & $\begin{array}{c}\text { Stress in } \\
\text { Y-direction } \\
\left(\mathrm{N} / \mathrm{mm}^{2}\right)\end{array}$ & $\begin{array}{c}\text { Von-mises } \\
\text { stress } \\
\left(\mathrm{N} / \mathrm{mm}^{2}\right)\end{array}$ \\
\hline 1 & Alloy steel & $0.52 \mathrm{e}-8$ & $0.30 \mathrm{e}-8$ & 23.09 & 24.73 & 28.76 \\
\hline 2 & $\begin{array}{c}\text { Titanium alloy, } \\
\text { Ti6al4V }\end{array}$ & $0.10 \mathrm{e}-8$ & $0.61 \mathrm{e}-8$ & 23.08 & 25.16 & 28.74 \\
\hline 3 & $\begin{array}{c}1044 \text { Carbon } \\
\text { steel }\end{array}$ & $0.53 \mathrm{e}-9$ & $0.31 \mathrm{e}-8$ & 23.08 & 24.86 & 28.75 \\
\hline 4 & $\begin{array}{c}\text { Aluminum } \\
\text { alloy, A6061 }\end{array}$ & $0.16 \mathrm{e}-8$ & $0.92 \mathrm{e}-8$ & 23.07 & 25.52 & 28.73 \\
\hline
\end{tabular}

Table 4.6 Compression loading at crank end (20.67 N/mm²) on Fig: 4.1 model

\begin{tabular}{|c|c|c|c|c|c|c|}
\hline S.No. & $\begin{array}{c}\text { Name of the } \\
\text { material }\end{array}$ & $\begin{array}{c}\text { Deformation in } \\
\text { X-direction } \\
(\mathrm{mm})\end{array}$ & $\begin{array}{c}\text { Deformation in } \\
\text { Y-direction } \\
(\mathrm{mm})\end{array}$ & $\begin{array}{c}\text { Stress in X- } \\
\text { direction } \\
\left(\mathrm{N} / \mathrm{mm}^{2}\right)\end{array}$ & $\begin{array}{c}\text { Stress in } \\
\text { Y- } \\
\text { direction } \\
\left(\mathrm{N} / \mathrm{mm}^{2}\right)\end{array}$ & $\begin{array}{c}\text { Von-mises } \\
\text { stress } \\
\left(\mathrm{N} / \mathrm{mm}^{2}\right)\end{array}$ \\
\hline 1 & Alloy steel & $0.11 \mathrm{e}-8$ & $0.62 \mathrm{e}-8$ & 47.21 & 50.56 & 58.80 \\
\hline 2 & $\begin{array}{c}\text { Titanium alloy, } \\
\text { Ti6al4V }\end{array}$ & $0.21 \mathrm{e}-8$ & $0.12 \mathrm{e}-9$ & 47.19 & 51.45 & 58.77 \\
\hline 3 & $\begin{array}{c}1044 \text { Carbon } \\
\text { steel }\end{array}$ & $0.11 \mathrm{e}-8$ & $0.63 \mathrm{e}-8$ & 47.21 & 50.84 & 58.79 \\
\hline 4 & $\begin{array}{c}\text { Aluminum alloy, } \\
\text { A6061 }\end{array}$ & $0.32 \mathrm{e}-8$ & $0.19 \mathrm{e}-7$ & 47.17 & 52.18 & 58.74 \\
\hline
\end{tabular}


Table 4.7 Tension loading at pin end $\left(22.24 \mathrm{~N} / \mathrm{mm}^{2}\right)$ on Fig: 4.1 model

\begin{tabular}{|c|c|c|c|c|c|c|}
\hline S.No. & $\begin{array}{c}\text { Name of the } \\
\text { material }\end{array}$ & $\begin{array}{c}\text { Deformation in } \\
\text { X- direction } \\
(\mathrm{mm})\end{array}$ & $\begin{array}{c}\text { Deformation in } \\
\text { Y- direction } \\
(\mathrm{mm})\end{array}$ & $\begin{array}{c}\text { Stress in } \\
\mathrm{X}- \\
\text { direction } \\
\left(\mathrm{N} / \mathrm{mm}^{2}\right)\end{array}$ & $\begin{array}{c}\text { Stress in } \\
\text { Y- } \\
\text { direction } \\
\left(\mathrm{N} / \mathrm{mm}^{2}\right)\end{array}$ & $\begin{array}{c}\text { Von- } \\
\text { mises } \\
\text { stress } \\
\left(\mathrm{N} / \mathrm{mm}^{2}\right)\end{array}$ \\
\hline 1 & Alloy steel & $0.40 \mathrm{e}-8$ & $0.34 \mathrm{e}-8$ & 46.95 & 60.02 & 62.44 \\
\hline 2 & $\begin{array}{c}\text { Titanium } \\
\text { alloy, Ti6al4V }\end{array}$ & $0.81 \mathrm{e}-8$ & $0.70 \mathrm{e}-8$ & 47.78 & 61.34 & 62.39 \\
\hline 3 & $\begin{array}{c}1044 \text { Carbon } \\
\text { steel }\end{array}$ & $0.41 \mathrm{e}-8$ & $0.35 \mathrm{e}-8$ & 47.21 & 60.42 & 62.43 \\
\hline 4 & $\begin{array}{c}\text { Aluminum } \\
\text { alloy, A6061 }\end{array}$ & $0.13 \mathrm{e}-7$ & $0.16 \mathrm{e}-7$ & 48.45 & 62.45 & 62.34 \\
\hline
\end{tabular}

Table 4.8 Compression loading at pin end $\left(20.17 \mathrm{~N} / \mathrm{mm}^{2}\right)$ on Fig: 4.1 model

\begin{tabular}{|c|c|c|c|c|c|c|}
\hline S.No. & $\begin{array}{c}\text { Name of the } \\
\text { material }\end{array}$ & $\begin{array}{c}\text { Deformation } \\
\text { in } \\
\text { X-direction } \\
(\mathrm{mm})\end{array}$ & $\begin{array}{c}\text { Deformation } \\
\text { in } \\
\text { Y- direction } \\
(\mathrm{mm})\end{array}$ & $\begin{array}{c}\text { Stress in } \\
\mathrm{X}- \\
\text { direction } \\
\left(\mathrm{N} / \mathrm{mm}^{2}\right)\end{array}$ & $\begin{array}{c}\text { Stress in } \\
\text { Y- } \\
\text { direction } \\
\left(\mathrm{N} / \mathrm{mm}^{2}\right)\end{array}$ & $\begin{array}{c}\text { Von- } \\
\text { mises } \\
\text { stress } \\
\left(\mathrm{N} / \mathrm{mm}^{2}\right)\end{array}$ \\
\hline 1 & Alloy steel & $0.36 \mathrm{e}-8$ & $0.31 \mathrm{e}-8$ & 42.58 & 54.43 & 56.65 \\
\hline 2 & $\begin{array}{c}\text { Titanium alloy, } \\
\text { Ti6al4V }\end{array}$ & $0.74 \mathrm{e}-8$ & $0.69 \mathrm{e}-8$ & 43.33 & 55.63 & 56.58 \\
\hline 3 & $\begin{array}{c}\text { 1044 Carbon } \\
\text { steel }\end{array}$ & $0.37 \mathrm{e}-8$ & $0.32 \mathrm{e}-8$ & 42.81 & 54.80 & 56.62 \\
\hline 4 & $\begin{array}{c}\text { Aluminum alloy, } \\
\text { A6061 }\end{array}$ & $0.11 \mathrm{e}-7$ & $0.96 \mathrm{e}-8$ & 43.94 & 56.64 & 56.54 \\
\hline
\end{tabular}

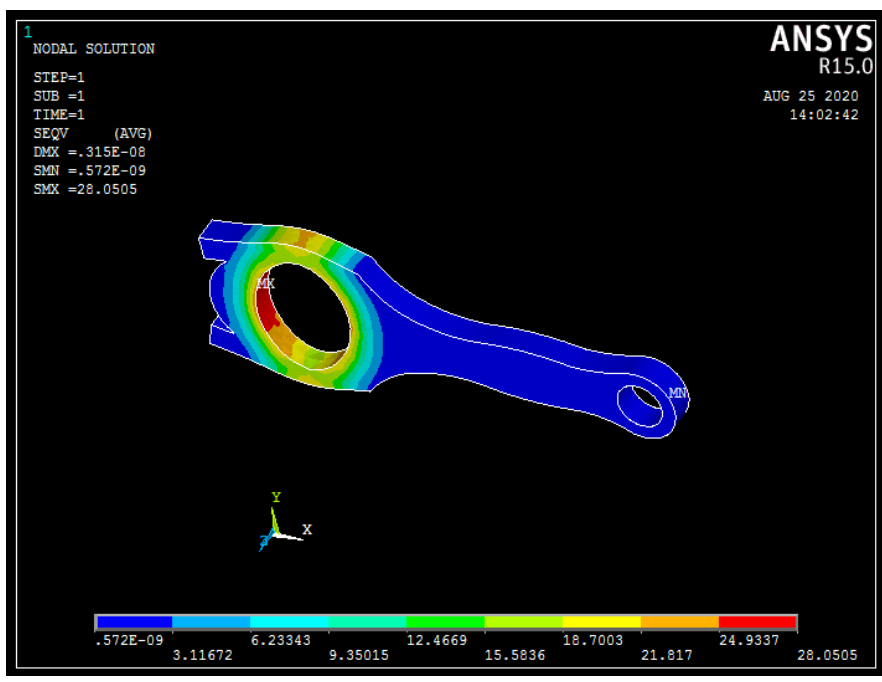

Fig: 4.2 Von-mises stress under tension loading at crank end $\left(10.11 \mathrm{~N} / \mathrm{mm}^{2}\right)$ Alloy steel model

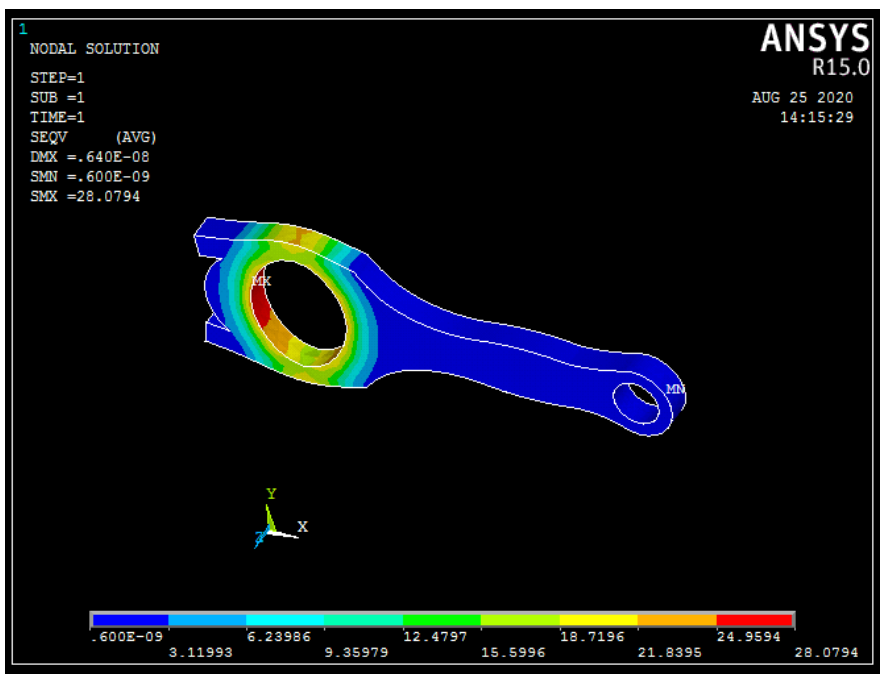

Fig: 4.3 Von-mises stress under tension loading at crank end $\left(10.11 \mathrm{~N} / \mathrm{mm}^{2}\right)$ Ti6Al4V model 


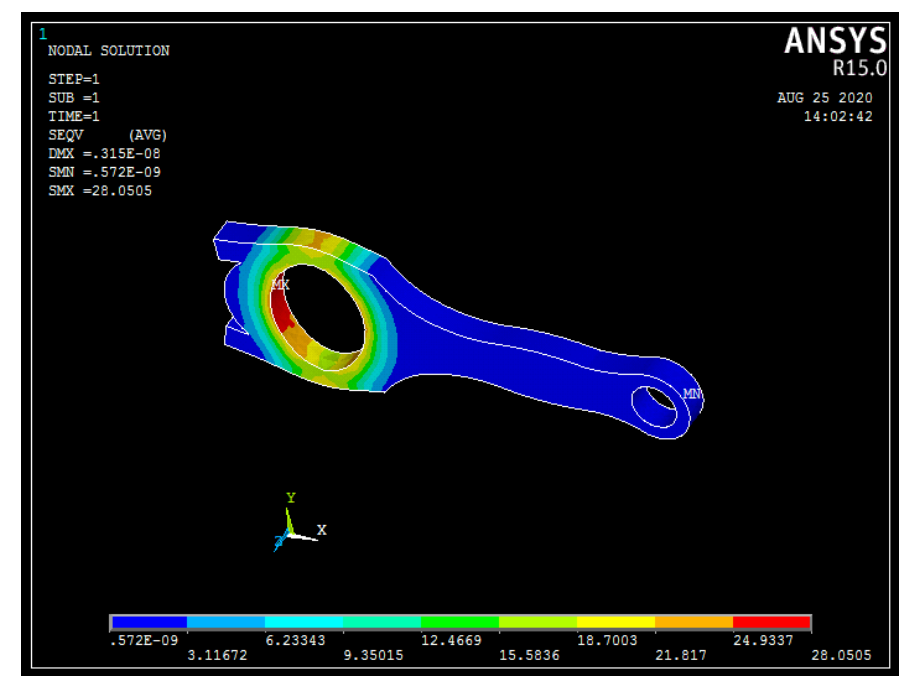

Fig: 4.4 Von-mises stress under tension loading at crank end $\left(10.11 \mathrm{~N} / \mathrm{mm}^{2}\right)$ Carbon steel model

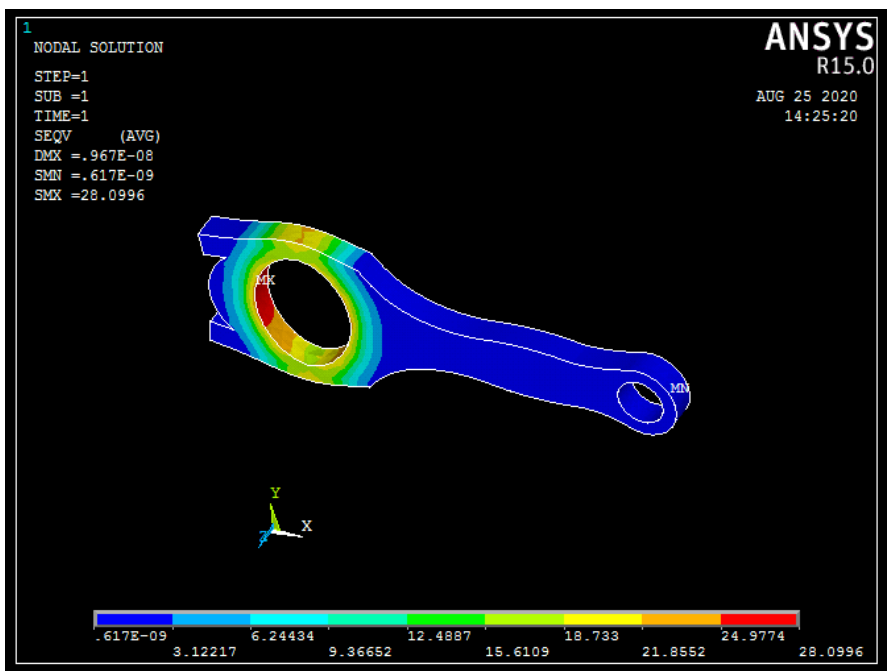

Fig: 4.5 Von-mises stress under tension loading at crank end $\left(10.11 \mathrm{~N} / \mathrm{mm}^{2}\right)$ Aluminum alloy model

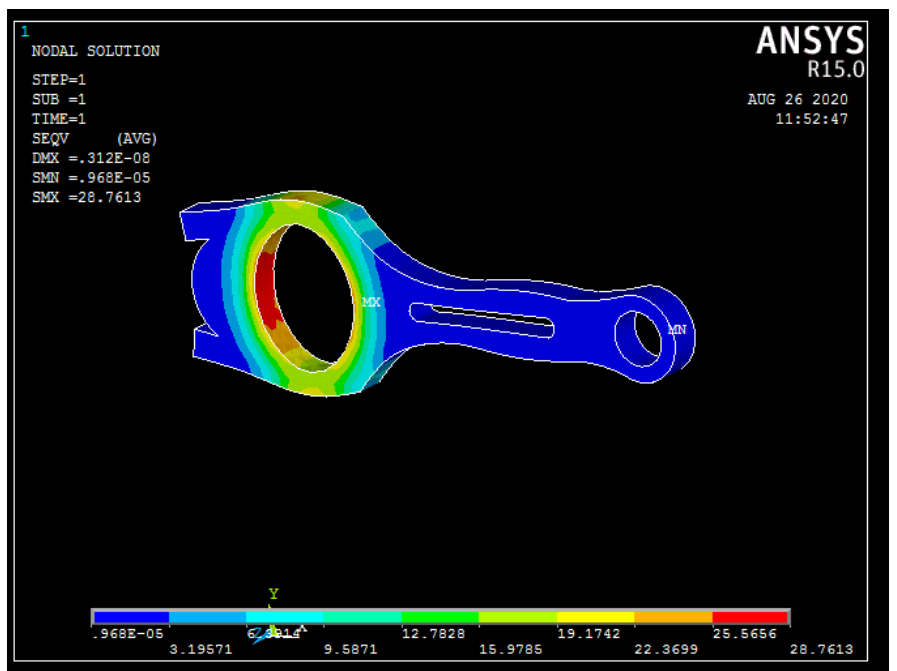

Fig: 4.5 Von-mises stress under tension loading at crank end $\left(10.11 \mathrm{~N} / \mathrm{mm}^{2}\right)$ Alloy steel optimized model

\section{CONCLUSIONS AND FUTURE SCOPE}

A noticeable $1.972 \%$ in weight reduction and a $2.53 \%$ increase in the von-mises stress due to shape optimization is observed. Even the deformations of Aluminum alloy material are more, and it can be ignored based on the low weight advantage. Further similar models can be analyzed for its Eigen buckling and modal analysis to find its natural and forced frequencies. Shape optimization is also can be enhanced based on the strength considerations. 


\section{REFERENCES}

[1] Prof. N.P.Doshi, Prof .N.K.Ingole, "Analysis of Connecting Rod Using Analytical and Finite Element Method", International Journal of Modern Engineering Research, Vol.3, Issue.1, Jan-Feb. 2013 pp-65-68.

[2] FEM analysis of connecting rod by R.Vozenilek,C.Scholz (The Technical University of Liberec,Halkova )

[3] Dynamic Load Analysis and Fatigue Behavior of Forged Steel vs. Powder Metal Connecting Rods by Adila Afzal and Pravardhan Shenoy (The University of Toledo 2003)

[4] Moon Kyu Lee,Hyungyil "Buckling sensitivity of connecting rod to the shank sectional area reduction original research article" Material and Design vol 31 Issue 6 Page 2796-2803

[5] Saharash khare,O.P.Singh "Spalling investigation of connecting rod original research article" Engineering Failure Analysis Vol 19,Jan 2012 page 77-86

[6] S.griza,F.Bertoni,G.zanon,A.Reguly "fatigue in engine connecting rod bolt due to forming laps original research article" Engineering failure Analysis Vol 16,issue 5 july 2009,page 1542-1548.

[7] R.J.yang, D.L Dewhirst "Shape optimization of connecting rod pin end using a generic model original research article" Finite element in analysis and design vol 11,issue 3 july 1992,page 257-264.

[8] https://en.wikipedia.org/wiki/Ansys 